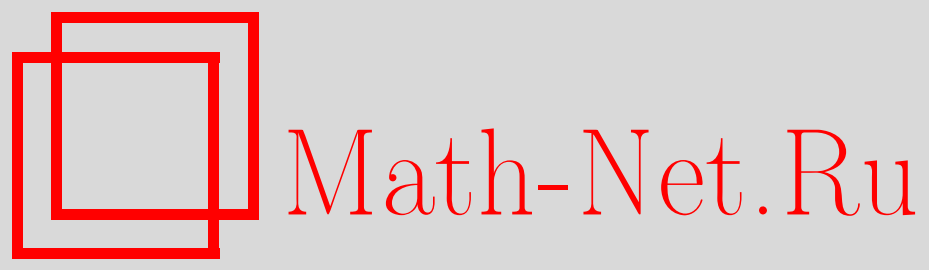

М. А. Красносельский, А. В. Лусников, Правильные неподвижные точки и устойчивые инвариантные множества монотонных операторов, Функи. анализ и его прил., 1996, том 30, выпуск $3,34-46$

DOI: https://doi.org/10.4213/faa536

Использование Общероссийского математического портала Math$\mathrm{Net.Ru}$ подразумевает, что вы прочитали и согласны с пользовательским соглашением http://www . mathnet.ru/rus/agreement

Параметры загрузки:

IP : 18.208 .226 .222

26 апреля 2023 г., $17: 15: 35$

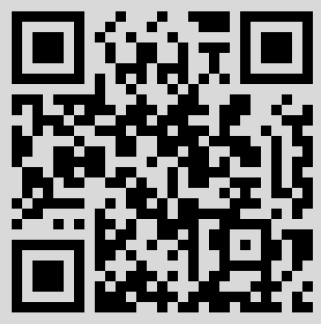


Функииональный анализ и его приложения

1996, т. 30, вып. 3, с. 34-46

УДК 517.96

\section{Правильные неподвижные точки и устойчивые инвариантные множества монотонных операторов}

(c) 1996. М. А. КРАСНоСЕЛЬСКИй, А. В. Лусников ${ }^{1}$

Предлагаются общие теоремы существования у разрывных операторов неподвижных точек с важными специальными свойствами и континуумов таких неподвижных точек. Используется терминология из [1-3].

\section{§1. Точки непрерывности}

Всюду ниже $E$ - это банахово пространство, полуупорядоченное конусом $K$. Используются обозначения: $x \leqslant y$, если $y-x \in K$, и $x<y$, если $x \leqslant y$ и $x \neq y$. Конус $K$ будем считать нормальным по М. Г. Крейну. Поэтому каждый конусный отрезок $\langle u, v\rangle=\{x \in E: u \leqslant x \leqslant v\}$ ограничен по норме, а из $0 \leqslant x \leqslant y$ вытекает оценка $\|x\| \leqslant N\|y\|$, где $N$ - константа нормальности конуса $K$. Действующий в $E$ оператор $A$ монотонен, если из $x \leqslant y$ следует $A x \leqslant A y$; он монотонно компактен, если из $x_{1} \leqslant \cdots \leqslant x_{n} \leqslant \cdots \leqslant w$ (из $x_{1} \geqslant$ $\cdots \geqslant x_{n} \geqslant \cdots \geqslant w$ ) вытекает сходимость по норме последовательности $A x_{n} \mathrm{k}$ некоторому элементу $z$, совпадающему с $\left\{\sup A x_{n}\right\}\left(\mathrm{c}\left\{\inf A x_{n}\right\}\right)$. Монотонный и монотонно компактный оператор назовем MMK-оператором.

Монотонную компактность монотонного оператора $A$ могут обеспечить различные причины. Достаточно, чтобы $A$ был компактным или конус $K$ обладал свойством правильности. Если $A=D G$, где $G$ монотонен как оператор из $E$ в банахово пространство $E_{1}$, полуупорядоченное правильным конусом $K_{1}$, то достаточно положительности действующего из $E_{1}$ в $E$ линейного непрерывного оператора $D$.

Оператор $A$ назовем $h$-монотонным, где $h \in K$ и $h \neq 0$, если из $x<y$ $(x, y \in E)$ вытекает соотношение $A x \leqslant A y-\alpha(x, y) h$, где $\alpha(x, y)>0$.

Точку $x^{*} \in E$ будем называть точкой $h$-непрерывности оператора $A$, если $\left\|A\left(x^{*}+\varepsilon h\right)-A x^{*}\right\| \rightarrow 0$ и $\left\|A\left(x^{*}-\varepsilon h\right)-A x^{*}\right\| \rightarrow 0$ при $\varepsilon \rightarrow+0$.

ТЕОРема 1. Пусть конусный отрезок $\langle u, v\rangle$ инвариантен для $h$-монотонного MMK-оператора $A$, причем $u<A u \leqslant A v<v$. Тогда у оператора $A$ есть по крайней мере одна неподвижная точка $x^{*} \in\langle u, v\rangle$, обладающая свойством $h$-непрерьвности.

ДоказАтЕльство. Определим оператор $C$ соотношением

$$
C x=\lim _{\varepsilon \rightarrow+0} A(x+\varepsilon h) .
$$

${ }^{1}$ Работа поддержана Российским фондом фундаментальных исследований, грант 93-0100884, и частично Международным научным фондом, грант MD4000. 
Предел (1) существует, так как $A$ является ММК-оператором. Из (1) следуют монотонность оператора $C$ и неравенства

$$
A x \leqslant C x \leqslant A(x+\varepsilon h) \quad(\varepsilon>0, x \in\langle u, v\rangle) .
$$

Сопоставим каждому $x \in\langle u, v\rangle$ множество

$$
S(x)=\left\{y \in\langle u, v\rangle: y \geqslant x, C y \geqslant y, C^{2} y>y+\delta(y) h, \delta(y)>0\right\} .
$$

Положим $\varphi(x)=\sup \left\{\left\|C^{2} y-y\right\|: y \in S(x)\right\}$, если $S(x)$ непусто, и $\varphi(x)=0$, если $S(x)$ пусто. Функция $\varphi(x)$ не возрастает: $\varphi(x) \leqslant \varphi(y)$ при $x>y$. Если $x \in S(u)$, то $\varphi(x)>0$.

Так как $u<A u$, то $u<C u$; поэтому из $h$-монотонности оператора $A$ вытекает, что $A(C u)>u+\delta(u) h$ и потому $C^{2} u>u+\delta(u) h$, где $\delta(u)>0$. Значит, $u \in S(u)$.

Построим монотонную последовательность $x_{1}=u, x_{n}=C^{2} t\left(x_{n-1}\right)$, где $t\left(x_{n-1}\right)$ - элемент множества $S\left(x_{n-1}\right)$, который удовлетворяет неравенству $\left\|C^{2} t\left(x_{n-1}\right)-t\left(x_{n-1}\right)\right\| \geqslant \varphi\left(x_{n-1}\right) / 2$. Для проверки корректности определения покажем, что из $t \in S(x)$ следует $C^{2} t \in S(x)$. Если $t \in S(x)$, то из (3) вытекает $C^{2} t>t+\delta(t) h$, где $\delta(t)>0$. Пусть $0<\varepsilon<\delta(t)$. Тогда в силу $h$-монотонности оператора $A$ при некотором $\delta_{*}>0$ справедлива оценка $A\left(C^{2} t\right)>A(t+\varepsilon h)+\delta_{*} h$ и, согласно $(2), C\left(C^{2} t\right)=C^{2}(C t) \geqslant C t+\delta_{*} h$. Неравенство $C(C t) \geqslant C t$ следует из (3) и монотонности оператора $C$. Поэтому элемент $C t$ принадлежит $S(x)$. Значит, и $C^{2} t \in S(x)$.

Из определения последовательности $x_{n}$ вытекает ее ограниченность сверху элементом $v$, а также существование таких $\delta_{n}>0$, что

$$
x_{n}+\delta_{n} h \leqslant x_{n+1} .
$$

Поэтому верна цепочка неравенств $x_{n} \leqslant C x_{n}<A\left(x_{n}+\delta_{n} h\right) \leqslant A x_{n+1}$, и из определения элементов $x_{n}$ следуют оценки

$$
A x_{n} \leqslant x_{n+1} \leqslant A x_{n+2} \quad(n=1,2, \ldots) .
$$

Последовательность $A x_{n}$ сходится, так как $A$ монотонно компактен. В силу (5) последовательность $x_{n}$ сходится к тому же пределу:

$$
\lim _{n \rightarrow \infty} x_{n}=\lim _{n \rightarrow \infty} A x_{n}=x^{*} \leqslant v .
$$

Числовая последовательность $\varphi\left(x_{n}\right)$ не возрастает и $\varphi\left(x_{n}\right) \rightarrow 0$; предположив противное, имеем $\varphi\left(x_{n}\right) \geqslant a>0$, откуда $\left\|t\left(x_{n}\right)-C^{2} t\left(x_{n}\right)\right\| \geqslant a / 2$ и $\left\|x_{n}-x_{n+1}\right\| \geqslant a / 2 N$, где $N$ - константа нормальности конуса $K$; последнее неравенство противоречит сходимости последовательности $x_{n}$. Так как функция $\varphi(x)$ не возрастает, то $\varphi\left(x^{*}\right)=0$. Из (6) следует, что $A x^{*} \geqslant x^{*}$ и поэтому $C x^{*} \geqslant x^{*}$. Допустим, что $C x^{*}>x^{*}$. Тогда $A\left(C x^{*}\right)>A x^{*}+\delta h$, где $\delta>0$. Отсюда и из $A x^{*} \geqslant x^{*}$ вытекает оценка $C^{2} x^{*}>x^{*}+\delta h$; поэтому из $C x^{*} \geqslant x^{*}$ следует $x^{*} \in S(u)$ и, далее, $\varphi\left(x^{*}\right) \geqslant\left\|C x^{*}-x^{*}\right\|>0$, что противоречит равенству $\varphi\left(x^{*}\right)=0$. Значит, $C x^{*}=x^{*}$, и $x^{*}-$ неподвижная точка оператора $A$; далее, $\left\|A\left(x^{*}+\varepsilon h\right)-x^{*}\right\| \rightarrow 0$ при $\varepsilon \rightarrow+0$. 
В силу соотношений (4) и (6) справедливы оценки $A x_{n}<A\left(x^{*}-\delta_{n} h\right)$. Поэтому $\left\|A\left(x^{*}-\varepsilon h\right)-x^{*}\right\| \rightarrow 0$ при $\varepsilon \rightarrow+0$. Теорема доказана.

Если конус $K$ телесен и $h \in \operatorname{int} K$, то точки $h$-непрерывности - это точки обычной непрерывности оператора $A$. Таким образом, теорема 1 (и последующие теоремы о точках $h$-непрерывности) отражает необычный и важный факт: в ее условиях разрывный оператор $A$ имеет неподвижные точки, в которых он непрерывен. Близкие к теореме 1 утверждения (в других условиях, другим методом и для других классов операторов) были получены в серии работ М. А. Красносельского и А. В. Покровского (см., например, [4-6]).

При изучении ряда задач, сводящихся к интегральным уравнениям

$$
x(t)=\int_{\Omega} K(t, s) f(s, x(s)) d s
$$

с разрывной по $x$ нелинейностью $f(s, x)$, особый интерес представляют решения $x_{*}(t)$, для которых функция $f\left(t, x_{*}(t)+v\right)$ почти при всех $t \in \Omega$ непрерывна по $v$ в точке $v=0$. Примером может служить известная задача М. А. Лаврентьева об отрывных течениях. Для ряда задач (включая проблему Лаврентьева) установлено существование решений с указанным свойством; эти решения названы правильными (см. [6]). Теорема 1 и последующие теоремы о точках $h$-непрерывности разрывного оператора $A$ в многочисленных конкретных приложениях приводят к признакам существования правильных решений; например, если $E$ - пространство скалярных или векторнозначных функций и для функции $h=h(t)$ выполняется равенство $\operatorname{mes}\{t \in \Omega: h(t)=0\}=0$, то в основных случаях $h$-устойчивые неподвижные точки являются правильными решениями уравнения $x=A x$.

В теореме 1 нет предположения о непрерывности оператора $A$. В связи с этим необходимо сказать несколько слов об общих уравнениях с разрывными операторами.

Пусть однозначный нелинейный и разрывный оператор $A$ действует в банаховом пространстве $E$. Тогда при изучении уравнения $x=A x$ обычно (см., например, $[7,8])$ используют его обобщенные решения; ими являются точки $x \in E$, для которых $x \in A^{0} x$, где $A^{0}$ - построенный по $A$ многозначный оператор. Ситуация меняется, если $E$ полуупорядочено, а оператор $A$ монотонен по полуупорядоченности. В этом случае могут быть указаны признаки существования обычных решений. Наиболее известный результат — теорема БиркгофаТарского [9], круг приложений которой ограничен, так как в ее условиях (как показывают примеры) каждое решение $x_{*}$ может быть точкой разрыва оператора $A$.

\section{§2. Устойчивость и робастная устойчивость}

Неподвижную точку $x^{*}$ монотонного оператора $A$ назовем $h$-устойчивой $(h>0)$, если существуют такие элементы $u_{n}, v_{n} \in E$ и числа $\delta_{n}>0, \sigma_{n}>0$, что

$$
u_{n}+\delta_{n} h<u_{n+1}<x^{*}<v_{n+1}<v_{n}-\sigma_{n} h \quad(n=1,2, \ldots)
$$


причем $u_{n} \leqslant A u_{n}<A v_{n} \leqslant v_{n},\left\|u_{n}-x^{*}\right\| \rightarrow 0$ и $\left\|v_{n}-x^{*}\right\| \rightarrow 0$. Назовем $h$-устойчивую неподвижную точку $x^{*}$ робастно $h$-устойчивой, если выполнено дополнительное условие

$$
u_{n} \leqslant A u_{n}-\mu_{n} h<x^{*}<A v_{n}+\mu_{n} h \leqslant v_{n}, \quad \mu_{n} \rightarrow+0 .
$$

Инвариантный для оператора $A$ конусный отрезок $\left\langle z_{*}, z^{*}\right\rangle \subset\langle u, v\rangle$ назовем особым, если $A z_{*}=z_{*}, A z^{*}=z^{*}$ и для каждого $x \in\left\langle z_{*}, z^{*}\right\rangle$ как из $A x \leqslant x$, так и из $A x \geqslant x$ вытекает равенство $A x=x$. Построение особых конусных отрезков является важным этапом исследования нелинейных операторов. Здесь рассматриваются монотонные операторы.

Конусный отрезок $\left\langle z_{*}, z^{*}\right\rangle \subset\langle u, v\rangle$ назовем робастно $h$-устойчивым для монотонного оператора $A$, если существуют $u_{n}, v_{n} \in\langle u, v\rangle$, для которых

$$
u_{1}<\cdots<u_{n}<\cdots<z_{*}, \quad z^{*}<\cdots<v_{n}<\cdots<v_{1},
$$

причем

$$
\begin{array}{cl}
\left\|u_{n}-z_{*}\right\| \rightarrow 0, & \left\|v_{n}-z^{*}\right\| \rightarrow 0, \\
u_{n}+\delta_{n} h \leqslant A u_{n} \leqslant u_{n+1}, & v_{n}-\delta_{n} h \geqslant A v_{n} \geqslant v_{n+1},
\end{array}
$$

где $\delta_{n}>0$ и $\delta_{n} \rightarrow 0$. Если $z_{*}=z^{*}$, то робастно $h$-устойчивый конусный отрезок вырождается в робастно $h$-устойчивую неподвижную точку $x^{*}$, являющуюся одновременно точкой $h$-непрерывности оператора $A$.

Теорема 2. В условиях теоремь 1 у оператора $A$ есть по крайней мере один робастно

ДокАЗАтЕльство. Сопоставим каждому элементу $x \in\langle u, v\rangle$ множество $M(x, v)=\{y: x \leqslant y<A y \leqslant v\}$; это множество при некоторых $x$ заведомо непусто, и, кроме того, $A y \in M(x, v)$ при каждом $y \in M(x, v)$. Положим

$$
\varphi(x, v)= \begin{cases}\sup \{\|A y-y\|: y \in M(x, v)\}, & \text { если } M(x, v) \text { непусто, } \\ 0, & \text { если } M(x, v) \text { пусто. }\end{cases}
$$

Функция $(11)$ не возрастает: $\varphi\left(x_{1}, v\right) \leqslant \varphi\left(x_{2}, v\right)$ при $x_{1} \leqslant x_{2}$. При этом из $\varphi(x, v)>0$ вытекает $\varphi(A x, v)>0$.

Пусть $x \in\langle u, v\rangle$ и $\varphi(x, v)>0$. Тогда существует элемент $g(x) \in M(x, v)$, для которого $\|A g(x)-g(x)\| \geqslant \varphi(x, v) / 2$.

Перейдем к построению последовательности $u_{n}$. Положим $u_{0}=u$ и $u_{n+1}=$ $A g\left(u_{n}\right)$. Так как $g\left(u_{1}\right) \leqslant \cdots \leqslant g\left(u_{n}\right) \leqslant \cdots \leqslant v$, то последовательность $u_{n}=$ $A g\left(u_{n-1}\right)$ сходится (в силу монотонной компактности оператора $A$ ) к некоторому элементу $z_{*}$. При этом $\varphi\left(u_{n}\right) \rightarrow 0$ и, значит, $\varphi\left(z_{*}\right)=0$. Отсюда вытекает, что $A z_{*}=z_{*}$.

Конусный отрезок $\left\langle z_{*}, v\right\rangle$ инвариантен для оператора $A$. Для построения последовательности $v_{n}$ применима конструкция, использованная выше при построении последовательности $u_{n}$. Нужно лишь вместо $M(x, v)$ использовать множества $M_{1}\left(x, z_{*}\right)=\left\{y: z_{*} \leqslant A y \leqslant y \leqslant x\right\}$ и вместо функции $\varphi(x, v)$ использовать функцию

$$
\varphi_{1}\left(x, z_{*}\right)= \begin{cases}\sup \left\{\|A y-y\|: y \in M_{1}\left(x, z_{*}\right)\right\}, & \text { если } M_{1}\left(x, z_{*}\right) \text { непусто, } \\ 0, & \text { если } M_{1}\left(x, z_{*}\right) \text { пусто }\end{cases}
$$


а затем определить при $x \in\left\langle z_{*}, v\right\rangle$ и $u_{1}\left(x, z_{*}\right)>0$ элемент $g_{1}(x) \in M_{1}\left(x, z_{*}\right)$, для которого $\left\|A g_{1}(x)-g_{1}(x)\right\| \geqslant \varphi_{1}\left(x, z_{*}\right) / 2$.

Очевидно, что для $u_{n}$ и $v_{n}$ выполнены соотношения $(9)-(10)$; кроме того, $z_{*} \leqslant z^{*}$. Так каK $\varphi\left(z_{*}, v\right)=0$ и $\varphi_{1}\left(z^{*}, z_{*}\right)=0$, то $\left\langle z_{*}, z^{*}\right\rangle$ является особым инвариантным для $A$ конусным отрезком. Теорема доказана.

ЗАмЕчАниЕ. Пусть ММК-оператор $A$ не обладает свойством $h$-монотонности, но $u<A u, A v<v$. Тогда также существует инвариантный для $A$ особый конусный отрезок $\left\langle z_{*}, z^{*}\right\rangle$ и сходящиеся соответственно к $z_{*}$ и $z^{*}$ последовательности $u=u_{0}<u_{1}<\cdots<u_{n}<\cdots<z_{*}$ и $v=v_{0}>v_{1}>\cdots>v_{n}>$ $\cdots>z^{*}$, причем $u_{n}<A u_{n} \leqslant z_{*}, v_{n}>A v_{n} \geqslant z^{*}, n=0,1, \ldots$.

Продолжим изучение ММК-операторов $A$.

Пусть $g \in K, g \neq 0$. Тогда при каждом $x$ определены скачки

$$
J(x, g,+0)=\lim _{\varepsilon \rightarrow+0} A(x+\varepsilon g)-A x, \quad J(x, g,-0)=A x-\lim _{\varepsilon \rightarrow+0} A(x-\varepsilon g)
$$

в точке $x$ оператора $A$ слева и справа по направлению $g$. Очевидно, что

$$
J(x, \alpha g,-0)=J(x, g,-0), \quad J(x, \alpha g,+0)=J(x, g,+0) \quad(\alpha>0) ;
$$

если $g_{1}>g$, то $J\left(x, g_{1},+0\right) \geqslant J(x, g,+0), J\left(x, g_{1},-0\right) \geqslant J(x, g,-0)$; далее, $J\left(x, g_{1},+0\right) \leqslant J\left(x, g_{1}+g_{2},+0\right), J\left(x, g_{1},-0\right) \leqslant J\left(x, g_{1}+g_{2},-0\right)$.

Часто сушествуют направления максимальных скачков. Если $K$ телесен, то каждый элемент $g \in \operatorname{int} K$ будет таким направлением. Если $K$ телесен и в точке $x$ максимальные скачки слева и справа равны нулю, то $x$ - обычная точка непрерывности. Если $K$ воспроизводящий, то для непрерывности монотонного оператора в точке $x$ достаточно равномерной непрерывности слева и справа по направлениям конуса.

Точка $x$ является точкой $h$-непрерывности оператора $A$, если и только если справедливы равенства $J(x, h,-0)=J(x, h,+0)=0$. Оператор $A$ назовем квалифицированно $h$-разрывным справа (слева), если из $J(x, h,+0)>0$ (из $J(x, h,-0)>0)$ вытекает существование таких $\beta>0,0<g<h$, что $J(x, g,+0)>\beta g$ (соответственно $J(x, g,-0)>\beta g)$. Квалифицированная $h$-разрывность многих конкретных операторов очевидна.

ТЕОРемА 3. Пусть выполнень условия теоремь 1. Пусть $z_{*} \neq z^{*} u$ $\left\langle z_{*}, z^{*}\right\rangle$ - робастно -устойчивый для $A$ конусный отрезок. Тогда из квалифицированной $h$-разрьвности справа (слева) оператора $A$ вытекает, что $z_{*}\left(\right.$ соответственно $z^{*}$ ) является неподвижной точкой оператора $A$, обладаюшей свойством $h$-непрерьвности.

ДокАзАтЕльство. Так как $\left\langle z_{*}, z^{*}\right\rangle$ робастно $h$-устойчив, то

$$
J\left(z_{*}, h,-0\right)=0, \quad J\left(z^{*}, h,+0\right)=0 .
$$

Поэтому из $z_{*}=z^{*}$ следует, что $z_{*}$ - точка $h$-непрерывности для $A$.

Пусть $z_{*} \neq z^{*}$. Если оператор $A$ квалифицированно $h$-разрывен справа ( $h$-разрывен слева), то в силу (12) достаточно показать, что $J\left(z_{*}, h,+0\right)=0$ (что $J\left(z^{*}, h,-0\right)=0$ ). Пусть для определенности $A$ квалифицированно $h$-разрывен справа и $J\left(z_{*}, h,+0\right) \neq 0$. Тогда $J\left(z_{*}, g,+0\right) \geqslant \beta g$, где $\beta>0$, $0<g<h$. Из $h$-монотонности оператора $A$ вытекают оценки

$$
A\left(z_{*}+\beta g\right) \geqslant A\left(z_{*}+\beta g / 2\right)+\gamma h \geqslant A z_{*}+J\left(z_{*}, g,+0\right)+\gamma h,
$$


где $\gamma>0$; поэтому $A\left(z_{*}+\beta g\right)>z_{*}+\beta g$. Так как $z_{*}+\beta g \in\left\langle z_{*}, z^{*}\right\rangle$, то отрезок $\left\langle z_{*}, z^{*}\right\rangle$ неособый. Полученное противоречие доказывает теорему.

ЗАмЕчАНИЕ. Если отбросить в условиях теоремы 3 предположение о квалифицированной $h$-разрывности оператора $A$, то можно лишь утверждать, что $A$ имеет в робастно $h$-устойчивом конусном отрезке $\left\langle z_{*}, z^{*}\right\rangle$ по крайней мере одну неподвижную точку со свойством $h$-непрерывности.

Теоремы об $h$-устойчивых, робастно $h$-устойчивых и просто устойчивых неподвижных точках важны не только при изучении импульсных динамических систем $x_{n+1}=A x_{n}$. Они применимы и при изучении различных дифференциальных и общих эволюционных уравнений. По поводу важного примера возможных приложений см., например, [10].

\section{§3. Континуумы неподвижных точек}

Через $\left\langle z_{1}, z_{2}\right\rangle$ ниже обозначаются конусные отрезки, инвариантные для оператора $A$, а через $B(z, r)$ - шар $\{x:\|x-z\| \leqslant r\}$. Через $F\left(z_{1}, z_{2}\right)$ обозначается множество $\left\{x: z_{1} \leqslant x \leqslant z_{2}, A x=x\right\}$.

Лемма 1. Пусть $\left\langle z_{*}, z^{*}\right\rangle$ - особьий для $h$-монотонного MМK-оператора А конусньй отрезок. Пусть в каждом особом для А конусном отрезке $\left\langle z_{1}, z_{2}\right\rangle \subset\left\langle z_{*}, z^{*}\right\rangle, z_{1}<z_{2}$, есть отличнье от $z_{1}, z_{2}$ точки $x_{*} \in F\left(z_{1}, z_{2}\right)$. Тогда $F\left(z_{*}, z^{*}\right)$ - это непрерьвная кривая

$$
\Gamma=\left\{x: x=\varphi(\alpha), \varphi(0)=z_{*}, \varphi(1)=z^{*}, 0 \leqslant \alpha \leqslant 1\right\},
$$

причем $\varphi(\alpha) \leqslant \varphi(\beta)-\delta(\alpha, \beta)$ при $\alpha<\beta$, где $\delta(\alpha, \beta)>0$.

ДокАЗАТЕЛЬСтво. Из $h$-монотонности $A$ вытекает, что достаточно установить равенство $F\left(z_{*}, z^{*}\right)=\Gamma$, где $\varphi(\beta)-\varphi(\alpha) \in K$ при $\alpha<\beta$.

Пусть $\left\langle z_{1}, z_{2}\right\rangle, z_{1}<z_{2}$, 一 особый конусный отрезок и $0<r<\left\|z_{2}-z_{1}\right\|$. Установим существование в $\left\langle z_{1}, z_{2}\right\rangle$ точки $x_{*} \in F\left(z_{1}, z_{2}\right)$, принадлежащей границе $\partial B\left(z_{1}, r\right)$ шара $B\left(z_{1}, r\right)$. Для этого сопоставим каждой точке $x \in G(r)=$ $F\left(z_{1}, z_{2}\right) \cap B\left(z_{1}, r\right)$ множество $Q(x)=\{y: y \geqslant x, y \in G(r)\}$. Определим на $Q\left(z_{1}\right)$ функцию

$$
\gamma(x)= \begin{cases}\sup \{\|y-x\|: y \in Q(x)\}, & \text { если } Q(x) \text { непусто, } \\ 0, & \text { если } Q(x) \text { пусто. }\end{cases}
$$

Построим последовательность $x_{n} \in F\left(z_{1}, z_{2}\right)$ по следующему правилу: $x_{1}=$ $z_{1}$; если элемент $x_{n-1}$ определен и $\gamma\left(x_{n-1}\right)=0$, то положим $x_{n}=x_{n-1}$; если $\gamma\left(x_{n-1}\right)=a>0$, то выберем такую точку $x_{n} \in Q\left(x_{n-1}\right)$, для которой $\left\|x_{n-1}-x_{n}\right\| \geqslant a / 2$. Последовательность $x_{n}=A x_{n}$ монотонна и поэтому сходится к некоторому пределу $x_{*}=A x_{*}$. Очевидно, что $x_{*} \in B\left(z_{1}, r\right)$ и $x_{*}<z_{2}$.

Для $x \in G_{1}(r)=F\left(x_{*}, z_{2}\right) \backslash B\left(z_{1}, r\right)$ положим $Q_{1}(x)=\{y: y \leqslant x, y \in$ $\left.G_{1}(r)\right\}$ и

$$
\gamma_{1}(x)= \begin{cases}\sup \left\{\|y-x\|: y \in Q_{1}(x)\right\}, & \text { если } Q_{1}(x) \text { непусто } \\ 0, & \text { если } Q_{1}(x) \text { пусто. }\end{cases}
$$

Определим элементы $w_{n}$ по правилу: $w_{1}=z_{2}$; если $\gamma_{1}\left(w_{n-1}\right)=0$, то $w_{n}=$ $w_{n-1}$; если $\gamma_{1}\left(w_{n-1}\right)=a>0$, то $w_{n} \in Q_{1}\left(w_{n-1}\right)$ и $\left\|w_{n}-w_{n-1}\right\| \geqslant a / 2$. Последовательность $w_{n}$ убывает и сходится к некоторой точке $w_{*}=A w_{*}$. Если 
$x_{*}<w_{*}$, то $\left\langle x_{*}, w_{*}\right\rangle$ - особый конусный отрезок и по условию леммы существует $z \in F\left(x_{*}, w_{*}\right), x_{*}<z<w_{*}$; существование такой точки $z$ противоречит построению одной из последовательностей $x_{n}, w_{n}$ в зависимости от принадлежности $z$ шару $B\left(z_{1}, r\right)$. Значит, $x_{*}=w_{*}$ и, далее, $x_{*} \in \partial B\left(z_{1}, r\right)$.

Показано, что каждому особому конусному отрезку $\left\langle z_{1}, z_{2}\right\rangle, z_{1}<z_{2}$, можно сопоставить точку $\psi\left(z_{1}, z_{2}\right) \in F\left(z_{1}, z_{2}\right)$, для которой

$$
\left\|\psi\left(z_{1}, z_{2}\right)-z_{1}\right\|=\left\|z_{1}-z_{2}\right\| / 2, \quad\left\|\psi\left(z_{1}, z_{2}\right)-z_{2}\right\| \geqslant\left\|z_{1}-z_{2}\right\| / 2 .
$$

Перейдем к построению кривой (13). Аргумент функции $\varphi(\alpha)$ будем писать в двоичной системе. Положим

$$
\begin{gathered}
\varphi(0)=z_{*}, \quad \varphi(1)=z^{*}, \quad \varphi(0,1)=\psi\left(z_{*}, z^{*}\right), \ldots, \\
\varphi\left(0, k_{1} \ldots k_{n} 0\right)=\varphi\left(0, k_{1} \ldots k_{n}\right) \\
\varphi\left(0, k_{1} \ldots k_{n} 1\right)=\psi\left(\varphi\left(0, k_{1} \ldots k_{n}\right), \varphi\left(0, k_{1} \ldots k_{n}+2^{-n}\right)\right), \ldots
\end{gathered}
$$

В точках $\alpha=0, k_{1} k_{2} \ldots k_{n} \ldots$, представимых только бесконечной двоичной дробью, определим $\varphi(\alpha)$ как предел монотонной и компактной последовательности $u_{n}=\varphi\left(0, k_{1} k_{2} \ldots k_{n}\right)$. Монотонность кривой $(13)$ очевидна, непрерывность следует из (15). Лемма доказана.

Пусть конус $K$ телесен и $h \in \operatorname{int} K$. Оператор $A$ назовем фокусирующим (cp. [11] для линейных $A$ ) на инвариантном для $A$ конусном отрезке $\langle u, v\rangle$, если он $h$-монотонен, если $u<A u<A v<v$ и, наконец, если найдется такое $\beta>0$, что

$$
B(A y-A x, 2 \beta\|A y-A x\|) \subset K \quad(u \leqslant x<y \leqslant v) .
$$

Число $\beta$ ниже предполагается малым. Каждый компактный фокусирующий на $\langle u, v\rangle$ оператор $A$ имеет в силу теоремы 2 особые инвариантные конусные отрезки $\left\langle z_{1}, z_{2}\right\rangle \in\langle u, v\rangle$. Положим $A_{n} x=A x+h / n$. Каждый оператор $A_{n}$ монотонен и компактен вместе с $A$. Выберем такое $n_{0}$, чтобы при $n \geqslant n_{0}$ была верна оценка $A_{n} A v<A v$. Тогда для оператора $A_{n}\left(n \geqslant n_{0}\right)$ инвариантен конусный отрезок $\left\langle z_{1}, A v\right\rangle$. Далее используется последовательность последовательностей $T_{n}=\left\{x: x=A_{n}^{m} z_{1}, m=0,1, \ldots\right\}$, где $n \geqslant n_{0}$.

ЛЕмма 2. В каждой последовательности $T_{n}$ есть лишь конечное число элементов особого отрезка $\left\langle z_{1}, z_{2}\right\rangle$.

ДокАЗАТЕльство. Так как последовательность $T_{n}$ монотонна и ограничена, то она сходится и $A_{n}^{m+1} z_{1}-A_{n}^{m} z_{1}=A A_{n}^{m} z_{1}-A_{n}^{m} z_{1}+h / n \rightarrow 0$ при $m \rightarrow \infty$. Поэтому $A A_{n}^{m} z_{1}<A_{n}^{m} z_{1}$ при больших $m$ и, так как конусный отрезок $\left\langle z_{1}, z_{2}\right\rangle$ особый, то $A_{n}^{m} z_{1} \notin\left\langle z_{1}, z_{2}\right\rangle$. Лемма доказана.

Tеорема 4. Пусть конус $K$ телесен $u h \in \operatorname{int} K$. Пусть оператор $A$ компактен и фокусирует на $\langle u, v\rangle$. Тогда построенное по робастно $h$-устойчивому особому конусному отрезку $\left\langle z_{*}, z^{*}\right\rangle$ (существуюшему в силу теоремь 2) множество $F\left(z_{*}, z^{*}\right)$ - это непрерьвная кривая (13), связьваюшая точки $z_{*} u z^{*}$.

ДокАЗАТЕЛЬСТво. В силу леммы 1 достаточно показать, что в каждом особом для $A$ конусном отрезке $\left\langle z_{1}, z_{2}\right\rangle \subset\left\langle z_{*}, z^{*}\right\rangle, z_{1}<z_{2}$, есть отличные от $z_{1}$ 
и $z_{2}$ неподвижные точки $x_{*}$ оператора $A$. Неподвижную точку $x_{*}$ будем искать в виде $x_{*}=C y_{*}$, где $z_{1}<y<z_{2}$, а $C$ - оператор (1).

В силу леммы 2 каждой последовательности $T_{n}$ можно сопоставить первый ее элемент $y_{n}=A_{n} x_{n}$, не принадлежащий шару $B\left(z_{1}, \beta\left\|z_{2}-z_{1}\right\|\right) \subset \frac{1}{2}\left(z_{1}+z_{2}\right)-K$. Тогда $x_{n} \leqslant\left(z_{1}+z_{2}\right) / 2$ и поэтому $A x_{n} \leqslant A\left(\left(z_{1}+z_{2}\right) / 2\right) \leqslant z_{2}-a h$, где $a>0$. Но $A z_{2}=z_{2}$ и, следовательно, $A x_{n} \leqslant z_{2}-a h$. Обозначим через $y_{*}$ предельную точку компактной последовательности $y_{n}=A x_{n}+\delta_{n} h \leqslant z_{2}-a h+h / n$. Тогда $y_{*} \leqslant z_{2}-a h$ и $y_{*}<z_{2}$; далее, $\left\|y_{*}-z_{1}\right\| \geqslant \beta\left\|z_{1}-z_{2}\right\|$, и, следовательно, $z_{1}<y_{*}$. Из неравенств $z_{1}<y_{*}<z_{2}$ и из $h$-монотонности оператора $A$ вытекает, что $z_{1}<C y_{*}<z_{2}$. Осталось установить равенство $A C y_{*}=C y_{*}$.

Оператор $A$ компактен, и поэтому можно предположить, что $y_{n(k)} \rightarrow y_{*}$ и $A y_{n(k)} \rightarrow w_{*}$ при $k \rightarrow \infty$, причем $n(k)<n(k+1)$. Так как $A y_{n(k)} \geqslant$ $y_{n(k)}-h / n(k)$, то $w_{*} \geqslant y_{*}$.

Поскольку конус $K$ телесен, каждому $\varepsilon>0$ отвечает такое $k_{0}(\varepsilon)$, что $y_{n(k)} \leqslant y_{*}+\varepsilon h$ и $A y_{n(k)} \leqslant A\left(y_{*}+\varepsilon h\right)$ при $k \geqslant k_{0}(\varepsilon)$. Следовательно, $y_{*} \leqslant$ $w_{*} \leqslant A\left(y_{*}+\varepsilon h\right)$. Переходя в последней оценке к пределу при $\varepsilon \rightarrow 0$, получаем неравенство $y_{*} \leqslant C y_{*}$. Поэтому $C^{2} y_{*} \geqslant C y_{*}$ и $0 \leqslant C^{2} y_{*}-C y_{*} \leqslant$ $A\left(C y_{*}+\delta h\right)-C y_{*}$ при любом $\delta>0$. Из последних оценок вытекает, что $\left\|C^{2} y_{*}-C y_{*}\right\| \leqslant N\left\|A\left(C y_{*}+\delta h\right)-C y_{*}\right\|$, где $N-$ константа нормальности конуса $K$. Следовательно,

$$
\frac{1}{N}\left\|C^{2} y_{*}-C y_{*}\right\| \leqslant\left\|A\left(y_{*}+\delta h\right)-C y_{*}\right\|+\left\|A\left(C y_{*}+\delta h\right)-A\left(y_{*}+\delta h\right)\right\| .
$$

Так как оператор $A$ фокусирует и $y_{*} \leqslant C y_{*}$, то при малых $\delta>0$ в силу (16)

$$
B\left(A\left(C y_{*}+\delta h\right)-A\left(y_{*}+\delta h\right), 2 \beta\left\|A\left(C y_{*}+\delta h\right)-A\left(y_{*}+\delta h\right)\right\|\right) \subset K .
$$

Поэтому из оценки

$$
\left\|A\left(y_{*}+\delta h\right)-\left(C y_{*}+\delta h\right)\right\|<\beta\left\|A\left(C y_{*}+\delta h\right)-A\left(y_{*}+\delta h\right)\right\|,
$$

которую можно записать в виде

$$
\begin{gathered}
\left\|\left[A\left(C y_{*}+\delta h\right)-A\left(y_{*}+\delta h\right)\right]-\left[A\left(C y_{*}+\delta h\right)-\left(C y_{*}+\delta h\right)\right]\right\| \\
<\beta\left\|A\left(C y_{*}+\delta h\right)-A\left(y_{*}+\delta h\right)\right\|,
\end{gathered}
$$

вытекает, что $A\left(C y_{*}+\delta h\right)-\left(C y_{*}+\delta h\right) \in \operatorname{int} K$. Но при малых $\delta>0$ элемент $C y+\delta h$ принадлежит особому для $A$ конусному отрезку $\left\langle z_{*}, z^{*}\right\rangle$ и поэтому неравенство $C y_{*}+\delta h<A\left(C y_{*}+\delta h\right)$ невозможно. Следовательно, при малых $\delta>0$ верна противоположная (18) оценка

$$
\left\|A\left(y_{*}+\delta h\right)-\left(C y_{*}+\delta h\right)\right\| \geqslant \beta\left\|A\left(C y_{*}+\delta h\right)-A\left(y_{*}+\delta h\right)\right\|,
$$

в силу которой из (17) вытекает, что

$$
\frac{1}{N}\left\|C^{2} y_{*}-C y_{*}\right\| \leqslant \frac{1+\beta}{\beta}\left\|A\left(y_{*}+\delta h\right)-C y_{*}\right\|+\frac{\delta}{\beta}\|h\| .
$$

Правая часть последнего неравенства стремится к нулю при $\delta \rightarrow+0$. Поэтому $C^{2} y_{*}=C y_{*}$, т. е. $C x_{*}=x_{*}$. Но $A x_{*} \leqslant C x_{*}$; значит, $A x_{*} \leqslant x_{*}$, и, так как отрезок $\left\langle z_{*}, z^{*}\right\rangle$ особый, то $A x_{*}=x_{*}$. Теорема доказана. 


\section{§4. Вторая теорема о континуумах неподвижных точек}

В следующей теореме к конусу $K$ предъявлено дополнительное требование; это позволяет ослабить требования к оператору $A$. Конус $K$ называют миниэдральным, если для каждых двух элементов $x, y \in K$ определены элементы $\sup (x, y), \inf (x, y)$.

ТеОРема 5. Пусть телесньй конус $K$ миниэдрален $u h \in \operatorname{int} K$. Пусть $\left\langle z_{*}, z^{*}\right\rangle, z_{*}<z^{*}$, - существующий в силу теоремы 2 робастно $h$-устойчивый особый для $h$-монотонного MMK-оператора $A$ конусный отрезок. Тогда $F\left(z_{*}, z^{*}\right)$ - это непрерьвнал кривал (13), свлзьввающал точки $z_{*} u z^{*}$.

ДокАЗАТЕЛЬСТво. В силу леммы 1 достаточно установить, что в каждом особом конусном отрезке $\left\langle z_{1}, z_{2}\right\rangle \subset\left\langle z_{*}, z^{*}\right\rangle, z_{1}<z_{2}$, есть точка $x_{*} \in F\left(z_{*}, z^{*}\right)$, отличная от $z_{1}$ и $z_{2}$. При построении точки $x_{*}$ будет использован вспомогательный оператор

$$
I x=\inf (A x, y), \quad z_{1} \leqslant x \leqslant y,
$$

где $y$ - фиксированный элемент из $\operatorname{int}\left\langle z_{1}, z_{2}\right\rangle$. Используемые ниже свойства оператора (19) выделим для удобства читателя в отдельную лемму.

Лемма 3. Пусть $\left\langle z_{1}, z_{2}\right\rangle \subset\left\langle z_{*}, z^{*}\right\rangle, z_{1}<z_{2},-$ особьй для $h$-монотонного MМK-оператора А конусный отрезок. Тогда справедливы следуюшие три утверждения:

(i) Oператор I лвляется MMK-оператором на $\left\langle z_{1}, y\right\rangle$.

(ii) Eсли $x \in\left\langle z_{1}, y\right\rangle$ u Ix $<x$, mo $\operatorname{Ix} \in M(x)$, где

$$
M(x)=\left\{\xi: z_{1} \leqslant I \xi<\xi<x\right\} .
$$

(iii) Если $x \in\left\langle z_{1}, y\right\rangle$ и Ix $<x$, то разность $y-x$ не являетсл внутренним элементом конуса $K$.

ДокаЗАТЕЛЬСТво. Монотонность оператора $I$ очевидна. Покажем, что этот оператор монотонно компактен на конусном отрезке $\left\langle z_{1}, z_{2}\right\rangle$. Рассмотрим монотонную последовательность $x_{n} \subset\left\langle z_{1}, y\right\rangle$; пусть для определенности она не возрастает. Тогда последовательность $A x_{n}$ также не возрастает и (так как оператор $A$ монотонно компактен) сходится к некоторому элементу $z \in\left\langle z_{1}, A y\right\rangle$. При этом $z \leqslant A x_{n}=z+\left(A x_{n}-z\right)$. Следовательно, $\inf (z, y) \leqslant I x_{n} \leqslant \inf (z, y)+A x_{n}-z$ и, далее,

$$
0 \leqslant I x_{n}-\inf (z, y) \leqslant A x_{n}-z \quad(n=1,2, \ldots) .
$$

Но $\left\|A x_{n}-z\right\| \rightarrow 0$ и поэтому $\left\|I x_{n}-\inf (z, y)\right\| \rightarrow 0$. Таким образом, $I$ монотонно компактен. Утверждение (i) леммы 3 доказано.

Для доказательства утверждения (ii) нужно показать, что $I^{2} x<I x$. Так как $I x<x$, то из $h$-монотонности оператора $A$ вытекает справедливость при некотором $\delta>0$ оценки

$$
A I x+\delta h<A x .
$$

Так как конус $K$ телесен, то $\alpha\left(y-I^{2} x\right) \leqslant \delta h$ при некотором $\alpha \in(0,1)$; поэтому из $(21)$ вытекает неравенство $I^{2} x+\alpha\left(y-I^{2} x\right) \leqslant A x$. Далее, в силу выпуклости конусного отрезка $\left\langle z_{1}, y\right\rangle$ верна оценка

$$
I^{2} x+\alpha\left(y-I^{2} x\right)=(1-\alpha) I^{2} x+\alpha y \leqslant y .
$$


Таким образом,

$$
I^{2} x+\alpha\left(y-I^{2} x\right) \leqslant \inf (A x, y)=I x,
$$

и так как $y-I^{2} x>0$, то $I^{2} x<I x$. Утверждение (ii) леммы 3 доказано.

Утверждение (iii) будем доказывать от противного. Допустим, что $y-x \in$ $\operatorname{int} K$. Тогда $y-I x \in \operatorname{int} K$ и поэтому $I x+\mu h \leqslant y$ при некотором $\mu>0$. Так как конус $K$ телесен, то $\nu(A x-I x) \leqslant y$ и из очевидной оценки $I x+\nu(A x-I x) \leqslant y$ вытекает, что

$$
I x+\nu(A x-I x) \leqslant \inf (A x, y)=I x .
$$

Значит, $I x=A x$. Но по условиям утверждения (iii) справедлива оценка $I x<x$. Таким образом, $A x<x$. Последнее неравенство невозможно, так как конусный отрезок $\left\langle z_{1}, z_{2}\right\rangle$ особый. Из полученного противоречия вытекает, что $y-x \notin$ int $K$. Утверждение (iii) леммы 3 доказано. Лемма полностью доказана.

Вернемся к доказательству теоремы 5 и перейдем к построению неподвижной точки $x_{*} \in\left\langle z_{1}, z_{2}\right\rangle$, отличной от $z_{1}$ и $z_{2}$. Если $A y=y$, то можно положить $x_{*}=y$. Остается рассмотреть более сложный случай, когда $A y \neq y$; так как конусный отрезок $\left\langle z_{1}, z_{2}\right\rangle$ является особым для $A$, то в этом случае элементы $y$ и $A y$ несравнимы и поэтому справедливы неравенства Iy $<y, I y<A y$.

Для построения неподвижной точки $x_{*}$ ниже параллельно конструируются последовательность $s_{n} \in\left\langle z_{1}, y\right\rangle$ и последовательность $x_{n}=I s_{n}$. Последовательность $s_{n}$ конструируется так, чтобы она не возрастала. Тогда, так как в силу утверждения (i) леммы 3 оператор $I$ монотонно компактен, последовательность $x_{n}$ будет сходиться к некоторой точке $x_{*}$. Для завершения доказательства останется показать, что $x_{*} \neq z_{1}, x_{*} \neq z_{2}$ и $A x_{*}=x_{*}$.

Положим $s_{1}=y, x_{1}=I s_{1}$. Согласно утверждению (ii) леммы $3, x_{1} \in M(y)$ и поэтому определено множество $M\left(x_{1}\right)$ (см. формулу (20)). Дальнейшее построение последовательностей $s_{n}, x_{n}$ проведем по индукции.

Пусть определены элементы $s_{1}, \ldots, s_{k}$ и элементы $x_{1}, \ldots, x_{k} \in M(y)$. Из $x_{k} \in M(y)$ вытекает, что множество $M\left(x_{k}\right)$ непусто. В качестве $s_{k+1}$ выберем в $M\left(x_{k}\right)$ такой элемент, для которого

$$
\left\|I s_{k+1}-s_{k+1}\right\| \geqslant \frac{1}{2} \sup \left\{\|I \xi-\xi\|: \xi \in M\left(x_{k}\right)\right\} \stackrel{\text { def }}{=} \chi_{k+1},
$$

а затем положим $x_{k+1}=I s_{k+1}$. По построению $s_{k+1}<s_{k}$ и $x_{k+1} \in M(y)$. Последовательности $s_{n}, x_{n}$ построены.

Напомним еще раз, что из монотонности и ограниченности последовательности $s_{n}$ вытекает в силу утверждения (i) леммы 3 сходимость последовательности $x_{n}=I s_{n}$ к некоторому элементу $x_{*}$. В силу того же утверждения (i) леммы 3 оператор $I$ монотонен; последовательность $x_{n}$ не возрастает и $x_{*} \leqslant x_{n}$. Следовательно, $I x_{*} \leqslant I x_{n}=I^{2} s_{n}$ и, согласно утверждению (ii) леммы $3, I x_{*} \leqslant x_{n}$ при всех $n$. Переходя в неравенстве $I x_{*} \leqslant x_{n}$ к пределу, получаем оценку

$$
I x_{*} \leqslant x_{*} .
$$

Допустим, что $I x_{*}<x_{*}$. Тогда $x_{*} \in M\left(x_{n}\right)$ при всех $n$ и потому

$$
2 \chi_{n} \geqslant\left\|I x_{*}-x_{*}\right\|>0 \quad(n=1,2, \ldots),
$$


где $\chi_{n}$ - числа, определенные в (22). Но так как $x_{n}<s_{n}<x_{n-1}$, то $0<$ $s_{n}-x_{n}<x_{n-1}-x_{n}$ и, следовательно,

$$
\left\|s_{n}-I s_{n}\right\|=\left\|s_{n}-x_{n}\right\| \leqslant N\left\|x_{n-1}-x_{n}\right\| \quad(n=2,3, \ldots) .
$$

Из сходимости последовательности $x_{n}$ к $x_{*}$ вытекает, что $\left\|x_{n-1}-x_{n}\right\| \rightarrow 0$ и, далее, $\left\|s_{n}-x_{n}\right\| \rightarrow 0$, т. е. справедливо равенство

$$
\lim _{n \rightarrow \infty}\left\|I s_{n}-s_{n}\right\|=0 .
$$

Но из (22) и (24) вытекает противоречащая (25) оценка

$$
\left\|I s_{n}-s_{n}\right\| \geqslant \frac{1}{2}\left\|I x_{*}-x_{*}\right\|>0 \quad(n=1,2, \ldots) .
$$

Из этого противоречия и из оценки $(23)$ следует равенство $I x_{*}=x_{*}$. Значит, $x_{*} \leqslant A x_{*}$, и так как $x_{*}$ принадлежит особому для $A$ конусному отрезку $\left\langle z_{1}, z_{2}\right\rangle$, то $A x_{*}=x_{*}$.

Неравенства $x_{*} \leqslant y<z_{2}$ очевидны, и осталось показать, что $z_{1} \neq x_{*}$.

Tак как $y-x_{n} \notin \operatorname{int} K$ при всех $n$ (в силу утверждения (iii) леммы 3 ), то $y-x_{*}$ также не является внутренней точкой конуса $K$. В то же время из определения элемента $y$ вытекает, что $y-z_{1} \in \operatorname{int} K$. Следовательно, $x_{*} \neq z_{1}$. Теорема 5 полностью доказана.

ЗАмЕчаниЕ. Пусть конус $K \subset E$ не обладает свойством телесности и $h>0$. Через $E_{h}$ обозначают (см. [11]) пространство элементов $x \in E$ с конечной нормой

$$
\|x\|_{h}=\inf \{\alpha:-\alpha\|h\| \leqslant x \leqslant \alpha\|h\|\} .
$$

Полуупорядочим $E_{h}$ телесным и нормальным конусом $K_{h}=K \cap E_{h}$; очевидно, что $h \in \operatorname{int} K_{h}$. Рассмотрим оператор $A=D G$. Здесь $G$ - оператор, действующий из $E_{h}$ в банахово пространство $E_{1}$, полуупорядоченное правильным конусом $K_{1}$, причем из $x<y$ следует $G x<G y$; линейный оператор $D: E_{1} \rightarrow E_{h}$ непрерывен, $h$-монотонен и положителен. Теоремы 4 и 5 дополняет вытекающее из теоремы 5 утверждение: если конус $K$ миниэдрален и $\left\langle z_{*}, z^{*}\right\rangle$ - особый робастно $h$-устойчивый для оператора $D G$ конусный отрезок, то множество $F(D G)$ всех лежащих в $\left\langle z_{*}, z^{*}\right\rangle$ неподвижных точек оператора $D G$ образует непрерывную кривую (13), связывающую $z_{*}$ и $z^{*}$.

В завершение раздела приведем приложения к проблеме существования устойчивых состояний равновесия.

Теорема 6. В условиях каждой из теорем 3 и 4 динамическая система $x_{n}=A x_{n-1}$ имеет в $\langle u, v\rangle$ по крайней мере одно устойчивое по Ляпунову состояние равновесия.

ДокАЗАТЕЛЬСТВО. Пусть $\left\langle z_{*}, z^{*}\right\rangle$-робастно $h$-устойчивый конусный отрезок, существующий в силу теоремы 2 . Если $z_{*}=z^{*}$, то в силу той же теоремы 2 состояние равновесия $z^{*}$ обладает свойством $h$-устойчивости, а значит, и свойством устойчивости по Ляпунову. Если $z_{*}<z^{*}$, то каждая точка кривой (13) будет $h$-устойчивым (и, следовательно, устойчивым по Ляпунову) состоянием равновесия для $A$, так как каждый конусный отрезок $\langle\varphi(\alpha), \varphi(\beta)\rangle$ инвариантен для оператора $A$. Теорема доказана. 


\section{§5. Неподвижные точки немонотонных операторов}

В этом разделе нормальный конус $K \subset E$ может не быть ни телесным, ни миниэдральным. Множество $F \subset E$ будем называть непрерывной ветвью, соединяющей точки $z_{*}, z^{*} \in E$, если $F$ имеет точки на границе $\partial \Omega$ каждой ограниченной области $\Omega$, содержащей одну и только одну из точек $z_{*}, z^{*}$.

ТЕОРема 7. Пусть конусный отрезок $\left\langle z_{*}, z^{*}\right\rangle$ является инвариантньм и особым для вполне непрерывного (не облзательно монотонного) оператора П. Тогда верны следующие утверждения:

(i) Множество $F(\Pi)=\left\{z: z_{*} \leqslant z \leqslant z^{*}, A z=z\right\}$ - это непрерьвная ветвь, соединяюшая точки $z_{*} u z^{*}$.

(ii) Если оператор П обладает свойством монотонности, то ветвь $F(\Pi)$ содержит непрерьвную кривую Г вида (13).

ДокАЗАТЕльство. Пусть $P$ - определенный на всем $E$ непрерывный проектор (см., например, [3]) на выпуклое множество $\left\langle z_{*}, z^{*}\right\rangle$. Оператор $P$ непрерывен и $P z=z$ при $z \in\left\langle z_{*}, z^{*}\right\rangle$.

Если утверждение (i) неверно, то найдется ограниченная область $\Omega \subset E$, на границе которой нет точек множества $F(\Pi)$ и которой принадлежит лишь одна из точек $z_{*}, z^{*}$. Рассмотрим векторные поля

$$
\begin{array}{ll}
\Phi(x, \lambda)=x-(1-\lambda) \Pi P x-\lambda z_{*} \quad & (x \in \partial \Omega, 0 \leqslant \lambda \leqslant 1), \\
\Psi(x, \lambda)=x-(1-\lambda) \Pi P x-\lambda z^{*} \quad & (x \in \partial \Omega, 0 \leqslant \lambda \leqslant 1) .
\end{array}
$$

Если все векторы $\Phi(x, \lambda), \Psi(x, \lambda)$ не равны нулю, то поля $\Phi(x, 1)=x-z_{*}$, $\Psi(x, 1)=x-z^{*}$ гомотопны полю $\Phi(x, 0) \equiv \Psi(x, 0) \equiv x-$ ПP $x$; поэтому вращения $\gamma_{1}, \gamma_{2}$ полей $x-z_{*}, x-z^{*}$ на $\partial \Omega$ одинаковы (см. [3]). Но одно из чисел $\gamma_{1}, \gamma_{2}$ равно нулю, а второе равно 1. Значит, хотя бы одно из полей $\Phi(x, \lambda), \Psi(x, \lambda)$ принимает нулевые значения.

Пусть для определенности $\Phi\left(x_{0}, \lambda_{0}\right)=x_{0}-\left(1-\lambda_{0}\right) \Pi P x_{0}-\lambda_{0} z_{*}=0$, где $x_{0} \in \partial \Omega, \lambda_{0} \in[0,1]$. Так как $\Pi P x_{0} \in\left\langle z_{*}, z^{*}\right\rangle$, то $z_{*}<x_{0}<z^{*}$ и $x_{0}=$ $\left(1-\lambda_{0}\right) \Pi x_{0}+\lambda_{0} z_{*}$. Следовательно, $x_{0}-\Pi x_{0}=\lambda_{0}\left(z_{*}-\Pi x_{*}\right) \leqslant 0$, т. е. $x_{0} \leqslant \Pi x_{0}$ и, так как $\left\langle z_{*}, z^{*}\right\rangle$ - это особый конусный отрезок для оператора $\Pi, x_{0}=\Pi\left(x_{0}\right)$. Значит, $x_{0} \in F(\Pi)$. Полученное противоречие доказывает утверждение (i).

Перейдем к утверждению (ii). Так как при любых $z_{1}, z_{2} \in F(\Pi), z_{1}<z_{2}$, конусный отрезок $\left\langle z_{1}, z_{2}\right\rangle$ является инвариантным и особым для оператора П, то на пересечении отрезка $\left\langle z_{1}, z_{2}\right\rangle$ со сферой $\left\{x:\left\|x-z_{1}\right\|=\left\|z_{2}-z_{1}\right\| / 2\right\}$ в силу утверждения (i) есть точки множества $F(\Pi)$. Остается провести рассуждения, использованные при доказательстве леммы 1. Теорема 7 доказана.

При выполнении настоящей работы авторы тщательно изучали докторскую диссертацию А. В. Покровского «Функциональные методы качественного анализа нелинейных систем управления» (ИПУ, Москва, 1989), содержащую важные нетривиальные идеи. Авторы благодарны Д. И. Рачинскому за обсуждение отдельных частей рукописи. 


\section{ЛИТЕРАТУРА}

1. Крейн М. Г., Рутман М. А. Линейные операторы, оставляющие инвариантным конус в пространстве Банаха. УМН, 3, вып. 1, 3-95 (1948).

2. Красносельский $M$. А. Положительные решения операторных уравнений. Наука, М. (1962).

3. Красносельский М. А., Забрейко П. Г. Геометрические методы нелинейного анализа. Наука, М. (1975).

4. Красносельский M. A., Покровский $A$. B. Правильные решения уравнений с разрывными нелинейностями. ДАН СССР, 226, №3, 506-509 (1976).

5. Pokrovskii A. Shuttle algorithm in investigation of systems with hysteresis nonlinearities. In: Models of Hysteresis, ed. A. Visintin, Pitman Res. Notes in Math. Ser., Vol. 286, Longman Sci. \& Tech. (1993), pp. 124-142.

6. Красносельский $M$. А., Покровский A. B. Об эллиптических уравнениях с разрывными нелинейностями. Доклады РАН, 342, № 6, 731-734 (1995).

7. Deimling K. Multivalued Differential Equations. Walter de Gruyter, Berlin-New York (1992).

8. Филиппов $A$. Ф. Дифференциальные уравнения с разрывными правыми частями. Наука, М. (1985).

9. Люстерник Л. А., Соболев В. И. Краткий курс функционального анализа. Наука, М. (1975).

10. Krasnoselsky M. A., Pokrovsky A. V., Semenov M. E. Stable periodic oscillations in systems with monotonous hysteresis nonlinearity. Proceedings of Equadiff, Vol. 7, Prague (1989), pp. 92-95.

11. Красносельский M. А., Ливиии E. A., Соболев А. В. Линейные позитивные системы. Наука, М. (1985).

Институт проблем

Поступило в редакцию

передачи информации

17 августа 1994 г. 